\section{(6) OPEN ACCESS}

\title{
Psychological distress, neuroticism, and cause-specific mortality: early prospective evidence from UK Biobank
}

\author{
G David Batty, ${ }^{1}$ Andrew M McIntosh, ${ }^{2}$ Tom C Russ, ${ }^{3}$ lan J Deary, ${ }^{3}$ Catharine R Gale ${ }^{3,4}$
}

\begin{abstract}
- Additional material is
published online only. To view please visit the journal online (http://dx.doi.org/10.1136/jech2016-207267).

${ }^{1}$ Department of Epidemiology and Public Health, University College London, London, UK

${ }^{2}$ Division of Psychiatry, University of Edinburgh, Edinburgh, UK

${ }^{3}$ Centre for Cognitive Ageing and Cognitive Epidemiology, University of Edinburgh, Edinburgh, UK

${ }^{4} \mathrm{MRC}$ Lifecourse Epidemiology Unit, University of Southampton, Southampton, UK
\end{abstract}

\section{Correspondence to}

Dr G David Batty, Department of Epidemiology and Public Health, University College London, London, 1-19 Torrington Place, London, UK, WC1E 6BT.E.; david.batty@ucl. ac.uk

Received 25 January 2016 Revised 16 June 2016 Accepted 3 July 2016 Published Online First 12 August 2016

\section{CrossMark}

To cite: Batty GD, Mclntosh AM, Russ TC, et al. J Epidemiol Community Health 2016;70:1136-1139.

\begin{abstract}
Background It is well established that psychological distress (depression and anxiety) is related to an increased risk of mortality. The personality trait of neuroticism, reflecting a relatively stable tendency towards negative emotions, has also been associated with elevated rates of death in some studies.

Accordingly, we tested the possibility that it is the neuroticism trait itself, rather than the distress state, that is generating an increased risk of mortality.

Methods We used data from the UK Biobank study, a UK-wide prospective cohort study (2006-2010) in which distress was ascertained using the Patient Health Questionnaire and neuroticism using the Eysenck Personality Questionnaire-Revised Short Form.

Results A mean of 6.2 years of follow-up of 308721 study members gave rise to 4334 deaths. Higher neuroticism was weakly associated with total mortality (age-adjusted and sex-adjusted HR per SD increase; $95 \%$ Cl $1.05 ; 1.02$ to 1.09 ), and moderately strongly correlated with distress symptoms $(r=0.55$, $p<0.0001)$. Distress symptoms were positively related to risk of total mortality (age-adjusted and sex-adjusted HR per SD increase in distress; $95 \% \mathrm{Cl} 1.23 ; 1.20$ to 1.26 ). This gradient was, in fact, slightly strengthened after adding neuroticism to the multivariable model (1.30; 1.26 to 1.34 ) but markedly attenuated after taking into account other covariates which included health behaviours and somatic disease $(1.16 ; 1.12$ to 1.20$)$. Similar results were apparent when cardiovascular disease, cancer and external cause of death were the end points of interest.
\end{abstract}

Conclusions While there was good a priori reasons to anticipate the neuroticism would at least partially explain the relation between distress symptoms and causespecific mortality, we found no such evidence in the present study.

\section{INTRODUCTION}

Individual-participant and literature-based meta-analyses reveal dose-response relationships between higher levels of psychological distress (depression/anxiety) and the risk of premature mortality and selected chronic diseases. ${ }^{1-3}$ These observations have led to the speculation that treatment for distress could usefully occur in individuals at lower levels of distress than is currently recommended. The personality trait of neuroticism, reflecting a relatively stable tendency towards negative emotions, has also been associated with elevated rates of death and cardiovascular disease (CVD) in some studies. ${ }^{4}$ These inter-relationships raise the possibility that it is the neuroticism trait itself, rather than the distress state, that is generating an elevated risk of mortality. With no empirical examination of this hypothesis, we assessed the impact of controlling for neuroticism on the distress-mortality relation alongside a series of more traditional explanatory variables, including health behaviours.

\section{METHODS}

UK Biobank, a UK-wide, on-going, prospective cohort study, has been described in detail. ${ }^{5}$ In brief, between 2006 and 2010, 502649 participants aged 37-73 years attended various geographically distributed research clinics. Members of the public visited an assessment centre completed a questionnaire, underwent an interview and took part in various physical assessments. Ethical approval was obtained from the National Health Service National Research Ethics Service and all participants providing written informed consent.

\section{Assessment of psychological distress and neuroticism}

Psychological distress was measured using the fouritem version of the Patient Health Questionnaire (PHQ-4). ${ }^{6}$ Items are rated on a four-point Likert scale from 0 (not at all) to 3 (nearly every day) such that possible total scores range from 0 to 12 (higher scores denote greater distress). Scores on the PHQ-4 show good agreement with longer scales, and correlate with demographic risk factors for depression and anxiety. ${ }^{7}$ Neuroticism was measured with the 12-item Eysenck Personality Questionnaire-Revised Short Form. ${ }^{8}$ Other covariate data were collected using standard protocols, including: health behaviours (smoking status, alcohol intake, physical activity, dietary characteristics), physical attributes (body mass index, systolic blood pressure, forced expiratory volume in 1 min, grip strength), existing disease (physician diagnoses of vascular or heart problems, diabetes, cancer, asthma, chronic lung disease, deep vein thrombosis or pulmonary embolism at baseline) and socioeconomic status (highest attained educational qualification).

Study participants were linked to the National Health Service's Central Registry at Southport, UK, which provided vital status data and, where applicable, cause of death. Having ascertained that the proportional hazards assumption had been met, we used Cox regression analyses with accompanying 95\% CIs to summarise the association between psychological distress and mortality experience. In our 
analyses, using the PHQ-4, psychological distress was categorised into three groups (score): $1(0), 2(1-2), 3(\geq 3)$. The selection of these categories was data driven: we wanted sufficient numbers of deaths in each distress category to conduct robust statistical analyses.

\section{RESULTS}

In online supplementary table $\mathrm{S} 1$, we show the relation between categories of the distress score and study member characteristics at baseline. In general, people with a higher distress score had a less favourable risk factor profile as evidenced by poorer health behaviours, a higher prevalence of chronic disease and elevated neuroticism scores $(\mathrm{r}=0.55, \mathrm{p}<0.0001)$. This was not a universal observation, however, in that levels of blood pressure and alcohol intake were somewhat lower in study members reporting a greater degree of distress.

A total of 308721 people (142983 women) had data on distress, neuroticism, other potential confounding variables and mortality. During a mean follow-up period of 6.2 years, 4334 people died. Table 1 shows the relation of distress scores with total and cause-specific mortality. Irrespective of the mortality outcome, there was a positive distress-death relationship such that an elevated mortality risk was apparent in people with higher distress scores. Higher neuroticism was weakly associated with total mortality (age-adjusted and sex-adjusted HR per SD (2.92) increase; 95\% CI 1.05; 1.02 to 1.09 ), however, in none of our analyses did controlling for neuroticism have an attenuating impact on the distress-mortality end point associations. Indeed, positive confounding, whereby the distressdisease relationship was strengthened, was generally apparent. In contrast, separate adjustment for each cluster of covariates led to partial attenuation, with the greatest impact apparent for health behaviours, irrespective of the outcome interest. Controlling simultaneously for an additional 15 covariates led to more marked attenuation of risk although a dose-response association remained, as we illustrate for total mortality in

Table 1 HRs $(95 \% \mathrm{Cl})$ for psychological distress in relation to cause-specific mortality: UK Biobank ( $N=308721)$

\begin{tabular}{|c|c|c|c|c|c|}
\hline & \multicolumn{3}{|c|}{ Distress categories } & \multirow{2}{*}{$\begin{array}{l}p \text { Value } \\
\text { for trend }\end{array}$} & \multirow[b]{2}{*}{ SD increase in distress } \\
\hline & $1(\mathrm{~N}=128505)$ & $2(\mathrm{~N}=112635)$ & $3(\mathrm{~N}=67581)$ & & \\
\hline \multicolumn{6}{|l|}{ Total (4334 deaths) } \\
\hline Age-adjusted and sex-adjusted & 1.0 (ref) & $1.22(1.14$ to 1.31$)$ & 1.77 (1.64 to 1.91$)$ & $<0.0001$ & 1.23 (1.20 to 1.26$)$ \\
\hline Age-adjusted, sex-adjusted and neuroticism-adjusted & 1.0 & 1.28 (1.19 to 1.38$)$ & 2.01 (1.84 to 2.20$)$ & $<0.0001$ & 1.30 (1.26 to 1.34$)$ \\
\hline Age-adjusted, sex-adjusted and health behaviours-adjusted & 1.0 & $1.16(1.09$ to 1.25$)$ & $1.49(1.38$ to 1.61$)$ & $<0.0001$ & $1.14(1.11$ to 1.18$)$ \\
\hline Age-adjusted, sex-adjusted and physical attributes-adjusted & 1.0 & 1.18 (1.10 to 1.27$)$ & 1.60 (1.48 to 1.72$)$ & $<0.0001$ & 1.18 (1.15 to 1.21$)$ \\
\hline Age-adjusted, sex-adjusted and existing disease-adjusted & 1.0 & 1.15 (1.07 to 1.23$)$ & 1.57 (1.45 to 1.69$)$ & $<0.0001$ & 1.18 (1.15 to 1.21$)$ \\
\hline Age-adjusted, sex-adjusted and SES-adjusted & 1.0 & 1.23 (1.15 to 1.32$)$ & $1.72(1.59$ to 1.85$)$ & $<0.0001$ & 1.21 (1.18 to 1.24$)$ \\
\hline Multiply-adjusted & 1.0 & 1.16 (1.08 to 1.25$)$ & 1.54 (1.40 to 1.68$)$ & $<0.0001$ & $1.16(1.12$ to 1.20$)$ \\
\hline \multicolumn{6}{|l|}{ Cancer (2807 deaths) } \\
\hline Age-adjusted and sex-adjusted & 1.0 (ref) & 1.19 (1.10 to 1.29$)$ & $1.59(1.44$ to 1.75$)$ & $<0.0001$ & $1.17(1.13$ to 1.21$)$ \\
\hline Age-adjusted, sex-adjusted and neuroticism-adjusted & 1.0 & 1.27 (1.16 to 1.36$)$ & 1.90 (1.69 to 2.12$)$ & $<0.0001$ & $1.25(1.20$ to 1.31$)$ \\
\hline Age-adjusted, sex-adjusted and health behaviours-adjusted & 1.0 & 1.14 (1.05 to 1.24$)$ & $1.38(1.25$ to 1.52$)$ & $<0.0001$ & 1.10 (1.07 to 1.14$)$ \\
\hline Age-adjusted, sex-adjusted and physical attributes-adjusted & 1.0 & 1.17 (1.07 to 1.27$)$ & 1.50 (1.36 to 1.65$)$ & $<0.0001$ & 1.14 (1.10 to 1.19$)$ \\
\hline Age-adjusted, sex-adjusted and existing disease-adjusted & 1.0 & 1.13 (1.04 to 1.23$)$ & 1.46 (1.32 to 1.61$)$ & $<0.0001$ & 1.14 (1.10 to 1.18$)$ \\
\hline Age-adjusted, sex-adjusted and SES-adjusted & 1.0 & 1.19 (1.09 to 1.30$)$ & $1.55(1.41$ to 1.70$)$ & $<0.0001$ & $1.16(1.12$ to 1.20$)$ \\
\hline Multiply-adjusted & 1.0 & 1.16 (1.07 to 1.28$)$ & 1.54 (1.37 to 1.72$)$ & $<0.0001$ & $1.15(1.10$ to 1.20$)$ \\
\hline \multicolumn{6}{|l|}{ Cardiovascular disease (890 deaths) } \\
\hline Age-adjusted and sex-adjusted & 1.0 (ref) & 1.25 (1.08 to 1.46$)$ & 1.85 (1.56 to 2.18$)$ & $<0.0001$ & 1.27 (1.20 to 1.34$)$ \\
\hline Age-adjusted, sex-adjusted and neuroticism-adjusted & 1.0 & 1.31 (1.12 to 1.54$)$ & 2.09 (1.71 to 2.55$)$ & $<0.0001$ & 1.34 (1.25 to 1.34$)$ \\
\hline Age-adjusted, sex-adjusted and health behaviours-adjusted & 1.0 & $1.17(1.00$ to 1.36$)$ & 1.40 (1.18 to 1.66$)$ & $<0.0001$ & 1.13 (1.07 to 1.20$)$ \\
\hline Age-adjusted, sex-adjusted and physical attributes-adjusted & 1.0 & 1.18 (1.01 to 1.37$)$ & 1.53 (1.29 to 1.82$)$ & $<0.0001$ & 1.18 (1.11 to 1.25$)$ \\
\hline Age-adjusted, sex-adjusted and existing disease-adjusted & 1.0 & 1.13 (0.97 to 1.32$)$ & 1.47 (1.24 to 1.75$)$ & $<0.0001$ & 1.17 (1.10 to 1.24$)$ \\
\hline Age-adjusted, sex-adjusted and SES-adjusted & 1.0 & 1.26 (1.08 to 1.47$)$ & 1.76 (1.48 to 2.08$)$ & $<0.0001$ & 1.24 (1.17 to 1.31$)$ \\
\hline Multiply-adjusted & 1.0 & 1.13 (0.96 to 1.32$)$ & $1.32(1.07$ to 1.65$)$ & 0.009 & $1.11(1.03$ to 1.20$)$ \\
\hline \multicolumn{6}{|l|}{ External (406 deaths) } \\
\hline Age-adjusted and sex-adjusted & 1.0 (ref) & 1.17 (0.92 to 1.48$)$ & 2.06 (1.62 to 2.62$)$ & $<0.0001$ & 1.31 (1.21 to 1.41$)$ \\
\hline Age-adjusted, sex-adjusted and neuroticism-adjusted & 1.0 & $1.16(0.91$ to 1.48$)$ & 2.01 (1.51 to 2.69$)$ & $<0.0001$ & 1.31 (1.20 to 1.34$)$ \\
\hline Age-adjusted, sex-adjusted and health behaviours-adjusted & 1.0 & 1.12 (0.88 to 1.41$)$ & $1.73(1.35$ to 2.21$)$ & $<0.0001$ & 1.22 (1.12 to 1.32$)$ \\
\hline Age-adjusted, sex-adjusted and physical attributes-adjusted & 1.0 & 1.13 (0.89 to 1.43$)$ & 1.85 (1.45 to 2.37$)$ & $<0.0001$ & $1.26(1.16$ to 1.36$)$ \\
\hline Age-adjusted, sex-adjusted and existing disease-adjusted & 1.0 & 1.09 (0.86 to 1.38$)$ & 1.77 (1.39 to 2.27$)$ & $<0.0001$ & 1.24 (1.15 to 1.35$)$ \\
\hline Age-adjusted, sex-adjusted and SES-adjusted & 1.0 & 1.17 (0.93 to 1.49$)$ & 1.97 (1.56 to 2.51$)$ & $<0.0001$ & $1.28(1.18$ to 1.38$)$ \\
\hline Multiply-adjusted & 1.0 & 1.06 (0.83 to 1.36$)$ & $1.53(1.14$ to 2.06$)$ & 0.009 & 1.17 (1.06 to 1.29$)$ \\
\hline
\end{tabular}




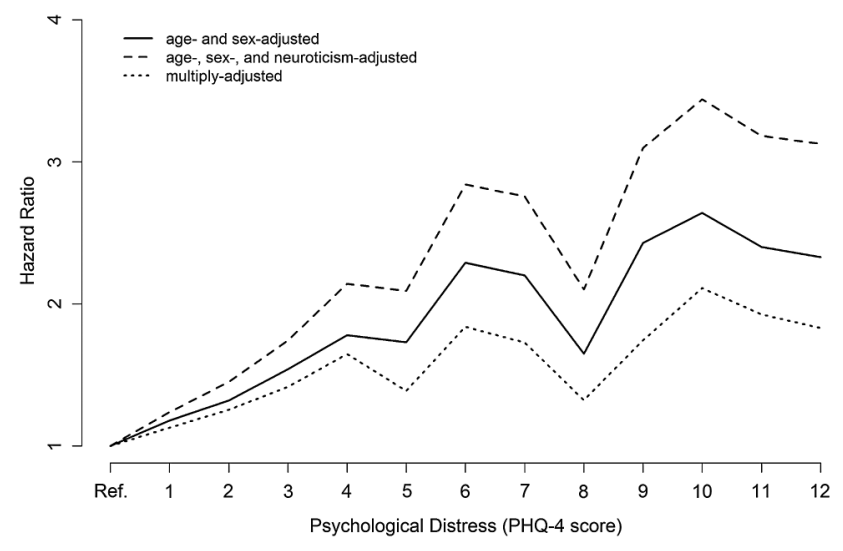

Figure 1 HRs for the full range of psychological distress scores in relation to total mortality: UK Biobank ( $\mathrm{N}=308$ 721). PHQ, Patient Health Questionnaire.

figure 1. Finally, we disaggregated the PHQ-4 into its four component parts (depressed mood, unenthusiasm/disinterest, tenseness/restlessness and tiredness/lethargy) and related these to total and CVD mortality (see online supplementary table S2). The same pattern of association was evident as for the total distress score.

\section{DISCUSSION}

We observed a dose-response relationship between a measure of psychological distress symptom severity and death from diseases of public health importance in men and women in a large, contemporary and well-characterised cohort study. Neuroticism, contrary to our prediction, had no explanatory power in this association.

\section{Study strengths and limitations}

We excluded 140352 participants from our analytical sample owing to missing data. Relative to the analytical sample, the excluded group had slightly higher scores for distress (mean 1.87 vs 1.49 ), and neuroticism (mean 3.78 vs 3.57 ), and higher mortality $(1.51 ; 1.45$ to 1.58$)$. The distress-total mortality relation (age-adjusted and sex-adjusted HR per SD increase in distress; 95\% CI) was also somewhat higher ( $\mathrm{p}$ value for interaction 0.009$)$ in the excluded group $(1.31 ; 1.27$ to 1.34$)$ than in the analytical sample $(1.23 ; 1.20$ to 1.26$)$, suggesting the HRs in our analytical sample may underestimate the true levels.

We assessed distress symptoms in the present study using a very brief inventory, the four-item PHQ, which was designed as a screening tool rather than a diagnostic device. Investigators on the largest other study to date to examine the distress-total mortality link administered a more detailed distress questionnaire, ${ }^{1}$ yet the age-adjusted and sex-adjusted HR per SD increase in distress $(1.21 ; 1.15$ to 1.27$)$ was almost identical to that found in the present study $(1.23 ; 1.20$ to 1.26$)$. Also in accordance with that study, here we found that the weakest relationship was apparent for cancer and the strongest for external causes of death. Additionally, the correlates of scores from the PHQ-4 in the present study-gender, health behaviours, somatic illness - are similar to those for depression and anxiety, suggesting some concurrent validity. Taken together, that a single administration of a very brief inventory of distress revealed relatively strong effects may be testimony to the robustness of these relationships.
Inevitably, we have failed to capture all confounding factors, or assessed crudely some of those included here. Residual confounding is a perennial concern in observational epidemiology that should, in principle, be circumvented by using the randomised controlled trial design. While it would be unethical to precipitate prolonged bouts of distress and observe effects on mortality, an alternative approach is to reverse depression using a pharmacological and/or spoken therapy and evaluate the impact of mortality experience, anticipating a lower risk in the intervention arm. In one of the few such trials conducted, based on a population of cardiac patients, people successfully treated for depression did not experience a reduction in event-free survival relative to the usual care group. ${ }^{9}$ In a cluster randomised trial of older people recruited from primary care settings, however, improved survival was, however, apparent in the treatment arm. ${ }^{10}$

Finally, the response proportion in UK Biobank, at $10 \%$, is very low by comparison with other studies. While this has impact on accurately estimating disease prevalence and incidence-any calculations are likely to be underestimates ${ }^{11}$-it has little implications for understating the aetiological role of risk factors for a given chronic disease for which UK Biobank was established. ${ }^{12}$ The original Whitehall study of Londonbased, non-industrialised civil servants (raised blood glucose as a risk factor for heart disease ${ }^{13}$ ), the British Doctors' study of registered physicians (cigarette smoking and selected cancers ${ }^{14}$ ) and Framingham study based in a single, affluent Massachusetts town (elevated serum cholesterol as a risk factor for heart disease $^{15}$ ) have all yielded findings of major public health importance despite being obviously unrepresentative of the general population. If a study has a large enough sample and succeeds in capturing the range of values within the exposure of interest, the results should be transportable. This logic has recently been supported by comparing results from an occupationally based study of civil servants (the second Whitehall study) with those from the geographically diverse British Regional Heart Study where near identical HRs across an array of known risk factors for coronary heart disease were reported. ${ }^{11}$ Similarly, in another cohort study, the relation of risk factor data collected at study induction to future CVD mortality was the same as that in a smaller, select group resurveyed 8 years later. ${ }^{16}$

\section{Plausible mechanisms}

That adjustment for an array of covariates, including neuroticism, did not eliminate the impact of distress on mortality risk inevitably raises speculation as to other mechanisms that may explain this relationship. Any such mechanisms are likely to be outcome-specific. Thus, bouts of acute anxiety may lead to acute coronary ischaemia that have been precipitated by coronary vasospasm ${ }^{17}$ and/or episodic elevations in blood pressure. Recurrent exposure to emotional disorder may also inhibit natural killer cell function which is implicated in immune system function, ${ }^{18}$ and therefore immunity-related cancers. The symptoms of fatigue, poor concentration and sleep disturbance, which characterise even moderately distressed individuals, may impact unfavourably on decision-making, risk perception, coordination and response time, so precipitating external causes of death such as accidents. ${ }^{19}$

In conclusion, while there was good a priori reasons to anticipate the neuroticism would at least partially explain the relation between distress symptoms and cause-specific mortality, we found no such evidence in the present study. 


\section{What is already known on this subject}

- Meta-analyses of observational studies have shown that psychological distress (depression and anxiety) is related to an increased risk of total mortality and cardiovascular disease.

- These gradients seem to be robust to control for various confounding factors, including health behaviours and socioeconomic status.

- The personality trait of neuroticism, reflecting a relatively stable tendency towards negative emotions, has been associated with elevated rates of death and cardiovascular disease in some studies.

\section{What this study adds}

- For the first time, to the best of our knowledge, we tested the possibility that it is the neuroticism trait itself, rather than the distress state, that is generating an elevated risk of mortality.

- Using data from UK Biobank, psychological distress was, as expected, associated with a range of mortality end points.

- We found no evidence supporting an explanatory role for neuroticism; rather, the greatest attenuating effect across multiple mortality outcomes was apparent for health behaviours.

Acknowledgements This research has been conducted using the UK Biobank Resource. TCR, GDB, CRG and IJD are members of the University of Edinburgh Centre for Cognitive Ageing and Cognitive Epidemiology, funding for which comes from the UK Biotechnology and Biological Sciences Research Council and the UK Medical Research Council. IJD and AMM are supported by the Wellcome Trust.

Contributors CRG, AMM, GDB were involved in study concept and design; CRG was involved in acquisition and preparation of the data set (including mortality linkage); CRG was involved in statistical analysis; all authors were involved in interpretation of the data; GDB and CRG were involved in drafting of the manuscript; all authors were involved in critical revision of the manuscript for important intellectual content. CRG had full access to all of the data in the study and takes responsibility for the integrity of the data and the accuracy of the data analysis. All authors saw and agreed on the final manuscript as well as the decision to submit for publication.

Competing interests None declared.

Ethics approval Ethical approval was obtained from the National Health Service National Research Ethics Service.

Provenance and peer review Not commissioned; externally peer reviewed.
Open Access This is an Open Access article distributed in accordance with the Creative Commons Attribution Non Commercial (CC BY-NC 4.0) license, which permits others to distribute, remix, adapt, build upon this work non-commercially, and license their derivative works on different terms, provided the original work is properly cited and the use is non-commercial. See: http://creativecommons.org/ licenses/by-nc/4.0/

\section{REFERENCES}

1 Russ TC, Stamatakis E, Hamer M, et al. Association between psychological distress and mortality: individual participant pooled analysis of 10 prospective cohort studies. BMJ 2012;345:e4933.

2 Pan A, Sun Q, Okereke Ol, et al. Depression and risk of stroke morbidity and mortality: a meta-analysis and systematic review. JAMA 2011;306:1241-9.

3 Batty GD, Russ TC, Stamatakis E, et al. Psychological distress and risk of peripheral vascular disease, abdominal aortic aneurysm, and heart failure: pooling of sixteen cohort studies. Atherosclerosis 2014;236:385-8.

4 Jokela M, Pulkki-Råback L, Elovainio M, et al. Personality traits as risk factors for stroke and coronary heart disease mortality: pooled analysis of three cohort studies. J Behav Med 2014;37:881-9.

5 Sudlow C, Gallacher J, Allen N, et al. UK biobank: an open access resource for identifying the causes of a wide range of complex diseases of middle and old age. PLoS Med 2015;12:e1001779.

6 Kroenke K, Spitzer RL, Williams JB, et al. An ultra-brief screening scale for anxiety and depression: the PHQ-4. Psychosomatics 2009;50:613-21.

7 Löwe B, Wahl I, Rose M, et al. A 4-item measure of depression and anxiety: validation and standardization of the Patient Health Questionnaire-4 (PHQ-4) in the general population. J Affect Disord 2010;122:86-95.

8 Deary IJ, Bedford A. Some origins and evolution of the EPQ-R (short form) neuroticism and extraversion items. Pers Individual Differences 2011;50:1213-17.

9 Berkman LF, Blumenthal J, Burg M, et al. Effects of treating depression and low perceived social support on clinical events after myocardial infarction: the Enhancing Recovery in Coronary Heart Disease Patients (ENRICHD) Randomized Trial. JAMA 2003:289:3106-16.

10 Gallo JJ, Morales KH, Bogner HR, et al. Long term effect of depression care management on mortality in older adults: follow-up of cluster randomized clinical trial in primary care. BMJ 2013;346:f2570.

11 Batty GD, Shipley M, Tabák A, et al. Generalizability of occupational cohort study findings. Epidemiology 2014;25:932-3.

12 Manolio TA, Collins R. Enhancing the feasibility of large cohort studies. JAMA 2010;304:2290-1.

13 Fuller JH, Shipley MJ, Rose G, et al. Coronary-heart-disease risk and impaired glucose tolerance. The Whitehall study. Lancet 1980;1:1373-6.

14 Doll R, Bradford Hill A. Mortality in relation to smoking: ten years' observations of British doctors. BMJ 1964;1:1399-410.

15 Kannel WB, Castelli WP, Revotskie N, et al. Profile of the coronary-prone individual. Assessment of risk of developing coronary heart disease. The Framingham study. Proc Annu Meet Med Sect Am Life Conv 1967;55:74-99.

16 Batty GD, Gale CR. Impact of resurvey non-response on the associations between baseline risk factors and cardiovascular disease mortality: prospective cohort study. J Epidemiol Community Health 2009;63:952-5.

17 Esler M, Alvarenga M, Lambert G, et al. Cardiac sympathetic nerve biology and brain monoamine turnover in panic disorder. Ann N Y Acad Sci 2004;1018: 505-14.

18 Kiecolt-Glaser JK, Robles TF, Heffner KL, et al. Psycho-oncology and cancer: psychoneuroimmunology and cancer. Ann Oncol 2002;13(Suppl 4):165-9.

19 Batty GD, Stamatakis E, Bell S. Psychological distress and risk of accidental death in the general population. Epidemiology 2016 